\title{
MODELLING OF BREAKING WAVES IN TSUNAMI AND SLOSHING WAVES BY A NEW PARTICLE METHOD*
}

\author{
Mimi Gao*, Chan Ghee $\mathrm{Koh}^{\dagger}$, Min Luo and Wei Bai ${ }^{\mathrm{It}}$ \\ National University of Singapore, Department of Civil and Environmental Engineering, \\ Singapore 117576, Singapore \\ “ceegaom@nus.edu.sg, †cgkoh@nus.edu.sg,
}

Published 17 November 2014

\begin{abstract}
The recently developed Consistent Particle Method (CPM) is used to model breaking waves in tsunami and violent sloshing waves in a moving tank. Solving the Navier-Stokes equations in a semi-implicit time stepping scheme, the CPM eliminates the use of kernel function which is somewhat arbitrarily defined and used in other particle methods. It is demonstrated that the method is applicable to large amplitude free surface wave problems that involve breaking phenomenon. Tsunami wave impact on a fixed structure is modeled using CPM. The simulated results show fairly good agreement to the actual nonlinear wave motions including overturning and breaking of waves. Large amplitude sloshing waves in a moving tank are investigated with CPM. Experiment was conducted in the laboratory to verify the CPM solutions. The hydrodynamic pressure computed by the CPM agrees well with the experimental results.
\end{abstract}

Keywords: Particle method; CPM; water sloshing; tsunami.

\section{Introduction}

Numerical simulation has made an enormous impact on the study of free surface motion of incompressible liquid such as liquid sloshing. Simulating this complex problem has many important applications, ranging from coastal protection and offshore structure design to LNG/oil sloshing on vessels. Nevertheless, these problems are difficult to simulate due to the free surface boundary condition is changing and not known a priori.

In recent years, a new generation of computational methods known as meshless methods is shown to outperform conventional mesh-based method in dealing with discontinuous fluid motion. Lagrangian meshless methods called particle methods have shown very good potential in dealing with large-amplitude free surface flows ${ }^{1}$, moving interfaces and deformable boundaries. The problem of numerical diffusion does not arise in particle methods ${ }^{2}$. The particle methods such as smooth particle hydrodynamics (SPH)

\footnotetext{
* This is an Open Access article published by World Scientific Publishing Company. It is distributed under the terms of the Creative Commons Attribution 3.0 (CC-BY) License. Further distribution of this work is permitted, provided the original work is properly cited.
} 
developed by Monaghan ${ }^{3,4}$ and moving-particle semi-implicit (MPS) method by Koshizuka and $\mathrm{Oka}^{5}$ have been used to solve fluid mechanic problems by many researchers ${ }^{1,2,6,7-11}$. Nevertheless, in SPH and MPS method, the approximation of partial differential operators requires a predefined kernel function. Accuracy is not necessarily satisfactory when the particle distribution is irregular. In particular, they tend to give severe and spurious pressure fluctuation.

In this study, a new particle method, called the Consistent Particle Method (CPM), is presented for large amplitude free-surface motion. The CPM computes the required partial differential operators in a way consistent with Taylor series expansion. A boundary particle recognition method is applied to help define the changing liquid domain. The incompressibility condition of free surface particles is enforced by an adjustment scheme. The CPM is used to model breaking waves in tsunami and violent sloshing waves in a moving tank. Liquid sloshing in prismatic tank is studied experimentally and numerically. The experimental results are used to validate the CPM in terms of free surface profile and dynamic pressure.

\section{Governing Equations}

The governing equations of viscous Newtonian fluid are expressed by the conservation laws of mass and momentum as follows:

$$
\begin{gathered}
\frac{1}{\rho} \frac{D \rho}{D t}+\nabla \cdot \mathbf{v}=0 \\
\frac{D \mathbf{v}}{D t}=-\frac{1}{\rho} \nabla p+v \nabla^{2} \mathbf{v}+\mathbf{g}
\end{gathered}
$$

where $\rho$ is the density of the fluid, $\mathbf{v}$ the particle velocity vector, $p$ the pressure, $v$ the kinematic viscosity and $\mathbf{g}$ the body force.

In the Consistent Particle Method, each fluid particle carries a mass $\mathrm{m}$ and moves at velocity $\mathbf{v}$. A particle interacts with its neighbouring particles with an influence radius $r_{e}$.

The proposed method involves a predictor-corrector algorithm comprising two steps the same as the MPS ${ }^{1}$ method. In the prediction step, the temporary particle velocities and positions are computed by considering only the body force and viscosity. In the correction step, Poisson's equation of pressure is used to enforce incompressibility. The pressure at new time step can be obtained by solving the Poisson's equation of pressure ${ }^{1}$ :

$$
\nabla^{2} p_{k+1}=-\frac{\rho}{(\Delta t)^{2}} \frac{\rho^{*}-\rho_{k+1}}{\rho_{k+1}}
$$

After applying appropriate discretization algorithm for the Laplace operator in Eq. (3), a system of linear equations can be obtained and solved using existing solvers such as incomplete Cholesky decomposition conjugate gradient method ${ }^{12}$ and GaussSeidel method ${ }^{9}$. Gauss-Seidel method is used in the present study. Particle number densities $n^{*}$ (corresponding to $\rho^{*}$ ) and $n_{0}$ (corresponding to $\rho_{0}$ ) are used in Eq. (3). 
Particle number density is a summation of the weight of each neighbour particle around a centre particle. The weights of the neighbours are computed the same as in Koshizuka et al. ${ }^{1}$

After the pressure term is obtained, the new particle velocities are computed (Eq. (4)) and the new positions of the particles are updated (Eq (5)). The time step is controlled in the computation to satisfy the Courant-Friedrichs-Lewy (CFL) condition ${ }^{6,13}$.

$$
\begin{aligned}
& \mathbf{v}_{k+1}=\mathbf{v}^{*}-\frac{\Delta t}{\rho} \nabla p_{k+1} \\
& \mathbf{r}_{k+1}=\mathbf{r}^{*}+\mathbf{v}_{k+1} \Delta t
\end{aligned}
$$

\section{Numerical algorithm}

\subsection{CPM formulation}

Given a differentiable functionf $(x, y)$, Taylor series expansion around a reference particle $\mathrm{P}\left(\mathrm{x}_{0}, \mathrm{y}_{0}\right)$ can be written in the form

$$
f(x, y)=f_{0}+h f_{, x 0}+k f_{, y 0}+\frac{1}{2} h^{2} f_{, x x 0}+h k f_{, x y 0}+\frac{1}{2} k^{2} f_{, y y 0}+O\left(r^{3}\right)
$$

where $h=x-x_{0}, k=y-y_{0}, f_{0}=f\left(x_{0}, y_{0}\right), f_{, x 0}=\frac{\partial f\left(x_{0}, y_{0}\right)}{\partial x}, f_{, y 0}=\frac{\partial f\left(x_{0}, y_{0}\right)}{\partial y}$, $f_{, x x 0}=\frac{\partial^{2} f\left(x_{0}, y_{0}\right)}{\partial x^{2}}, f_{, x y 0}=\frac{\partial^{2} f\left(x_{0}, y_{0}\right)}{\partial x \partial y}, f_{, y y 0}=\frac{\partial^{2} f\left(x_{0}, y_{0}\right)}{\partial y^{2}}, r=\sqrt{h^{2}+k^{2}}$.

If the values of $\mathrm{f}\left(\mathrm{x}_{\mathrm{j}}, \mathrm{y}_{\mathrm{j}}\right)$ are known at sufficient neighbouring particles $j(j=1,2 \ldots \mathrm{N})$, the series truncated to second order can be used to approximate the derivatives at the reference particle by solving a system of linear equations ${ }^{14}$.

Writing Eq. (6) for each of the neighbouring particles we get a set of linear equations

$$
[\mathbf{A}]\{D \mathbf{f}\}-\{\mathbf{f}\}=0
$$

The minimum number of neighbour particles required in Eq. (7) to determine $\{\mathrm{Df}\}$ vector is five since there are five unknowns. More than five particles should be used in order to improve the accuracy of the approximation ${ }^{14}$. In this case, an over-determined system of linear equations is solved by minimizing the following residual error

$$
\|\mathbf{E}\|=\sum_{j=1}^{N}\left[f_{0}-f_{j}+h_{j} f_{, x 0}+k_{j} f_{, y 0}+0.5 h_{j}^{2} f_{, x x 0}+h_{j} k_{j} f_{, x y 0}+0.5 k_{j}^{2} f_{, y y 0}\right]^{2} w_{j}^{2}
$$

where $w_{j}$ is the weighting function used in the least-square solution to account for the influence of distance. In this study, the weighting function proposed by Liszka and Orkisz $^{14}$ is used.

By minimizing the norm of $\mathbf{E}$, a set of equations is obtained, solving which leads to 


$$
\left\{\begin{array}{l}
f_{, x 0} \\
f_{, y 0} \\
f_{, x x 0} \\
f_{, x y 0} \\
f_{, y y 0}
\end{array}\right\}=\left[\begin{array}{lllll}
a_{1} & a_{2} & a_{3} & a_{4} & a_{5} \\
b_{1} & b_{2} & b_{3} & b_{4} & b_{5} \\
c_{1} & c_{2} & c_{3} & c_{4} & c_{5} \\
d_{1} & d_{2} & d_{3} & d_{4} & d_{5} \\
e_{1} & e_{2} & e_{3} & e_{4} & e_{5}
\end{array}\right]\left\{\begin{array}{c}
\sum f_{j} w_{j}^{2} h_{j}-f_{0} \sum w_{j}^{2} h_{j} \\
\sum f_{j} w_{j}^{2} k_{j}-f_{0} \sum w_{j}^{2} k_{j} \\
\sum f_{j} w_{j}^{2} \frac{h_{j}^{2}}{2}-f_{0} \sum w_{j}^{2} \frac{h_{j}^{2}}{2} \\
\sum f_{j} w_{j}^{2} h_{j} k_{j}-f_{0} \sum w_{j}^{2} h_{j} k_{j} \\
\sum f_{j} w_{j}^{2} \frac{k_{j}^{2}}{2}-f_{0} \sum w_{j}^{2} \frac{k_{j}^{2}}{2}
\end{array}\right\}
$$

where coefficients $a_{1}$ to $e_{5}$ depend only on the relative coordinates between the reference particle and its neighbour particles. Eq. (3) can be written as

$$
\begin{aligned}
\left(\nabla^{2} p\right)_{i}= & \sum_{j=1}^{N}\left\{w _ { j } ^ { 2 } \left[\left(c_{1}+e_{1}\right) h_{j}+\left(c_{2}+e_{2}\right) k_{j}+\left(c_{3}+e_{3}\right) \frac{h_{j}^{2}}{2}\right.\right. \\
& \left.\left.+\left(c_{4}+e_{4}\right) h_{j} k_{j}+\left(c_{5}+e_{5}\right) \frac{k_{j}^{2}}{2}\right]\right\}\left(p_{j}-p_{i}\right)=-\frac{\rho}{(\Delta t)^{2}} \frac{\rho^{*}-\rho^{0}}{\rho^{0}}
\end{aligned}
$$

Incompressibility in the fluid domain is satisfied by keeping the number density constant. The viscosity term in Eq. (2) is also approximated based on the same algorithm. The gradient model of pressure based on the solution of first derivatives becomes

$$
\begin{aligned}
(\nabla p)_{i x} & =\sum_{j=1}^{N}\left[w_{j}^{2}\left(a_{1} h_{j}+a_{2} k_{j}+a_{3} \frac{h_{j}^{2}}{2}+a_{4} h_{j} k_{j}+a_{5} \frac{k_{j}^{2}}{2}\right)\right]\left(p_{j}-p_{i}^{\prime}\right) \\
(\nabla p)_{i y} & =\sum_{j=1}^{N}\left[w_{j}^{2}\left(b_{1} h_{j}+b_{2} k_{j}+b_{3} \frac{h_{j}^{2}}{2}+b_{4} h_{j} k_{j}+b_{5} \frac{k_{j}^{2}}{2}\right)\right]\left(p_{j}-p_{i}^{\prime}\right)
\end{aligned}
$$

where $\mathrm{p}_{\mathrm{i}}^{\prime}=\min \left(\mathrm{p}_{\mathrm{j}}\right)$ for $\left\{\mathrm{j} \mid \mathrm{w}\left(\left|\mathrm{r}_{\mathrm{j}}-\mathrm{r}_{\mathrm{i}}\right|\right) \neq 0\right\}$.

Though the pressure value of any neighbour particle can be used for $p_{i}$ if the distribution of neighbour particles is isotropic, $\mathrm{p}_{\mathrm{i}}^{\prime}$ defined above is used instead of $\mathrm{p}_{\mathrm{i}}{ }^{1}$ This reduces the possibility of local congregation of fluid particles and hence greatly improves the stability of the algorithm. The stability improvement is very significant in moving boundary problems, such as fluid flows with free surface.

\subsection{Improved CPM with hybrid source term}

In the original CPM, fluid incompressibility is imposed by keeping the fluid density constant, which is called the zero-density-variation condition ${ }^{15}$. Alternatively, some studies ${ }^{15-17}$ enforces the incompressibility condition by projecting the intermediate fluid velocity onto a divergence-free space. Taking divergence of Eq. (4) gives

$$
\nabla \cdot \mathbf{v}_{k+1}=\nabla \cdot \mathbf{v}^{*}-\frac{\Delta t}{\rho} \nabla^{2} p_{k+1}
$$

Imposing the velocity-divergence-free condition $\nabla \cdot \mathbf{v}_{k+1}=0$, Eq. (13) is simplified to

$$
\nabla^{2} p_{k+1}=\frac{\rho}{\Delta t} \nabla \cdot \mathbf{v}^{*}
$$


Eqs. (3) and (14) are two different numerical techniques to enforce the incompressibility condition. Theoretically they are equivalent, but numerically their performances may be different. Using the zero-density-variation condition alone as Eq. (3), fluid volume can be conserved well and simulations are generally very stable, but there may be significant spurious pressure fluctuation ${ }^{17}$. On the other hand, the velocity divergence in Eq. (14) is less sensitive to errors of particle positions and therefore spurious fluctuation in pressure is mitigated ${ }^{18}$. Nevertheless, fluid volume may not be conserved well especially after a long-time simulation. Our study shows that the advantages of both approaches can be exploited by a hybrid scheme which combines the good features of both techniques through a weighting value $\gamma$ as shown in Eq. (15):

$$
\nabla^{2} p_{k+1}=\gamma \frac{\rho}{\Delta t^{2}} \frac{\rho_{0}-\rho^{*}}{\rho_{0}}+(1-\lambda) \frac{\rho}{\Delta t} \nabla \cdot \mathbf{v}^{*}
$$

where $\gamma$ is a parameter (between 0 and 1) to account for the relative contributions of the two terms. That is, with larger $\gamma$, the zero density-variation condition contributes more to the equation. $\gamma=0.1$ is found to be the optimum value for violent wave breaking cases in present study. Eq. (15) is called the pressure Poisson equation with a hybrid source term. For simplicity, the improved CPM with $\gamma$ value is denoted by CPM- $10 \times \gamma$. For example, CPM with $\gamma=1$ is denoted by CPM-10 (i.e. original CPM).

\subsection{Boundary surface recognition}

A key consideration is the effective recognition of free surface particles. The boundary particles should be recognized before the boundary conditions can be implemented. The MPS method uses density threshold to differentiate the free surface particles, particles satisfying $(n *)_{i}<\beta n_{0}$ are considered free surface particles. This simple threshold method is, however, ineffective in recognizing free surface particles accurately. It will cause great pressure errors when some inner fluid particles are falsely recognized as free surface particles. To tackle this problem, several boundary recognition algorithms are proposed ${ }^{19-}$ ${ }^{23}$. Dilts ${ }^{24}$ introduced a method called the "arc" method for fast and accurate boundary recognition. The arc method is used in present study.

\subsection{Incompressibility for free surface particles}

Incompressibility is imposed by Eq. (3), which corrects the temporary particle number densities to the constant $n_{0}$. The number density of free surface particles is less than that of the in-domain fluid particles due to the absence of particles above the free surface. Hence, the correction of number density to $\mathrm{n}_{0}$ is not applicable to free surface particles.

A scheme is proposed by Koh et al. ${ }^{25}$ to impose incompressibility for free surface particles. If the two free surface particles get too close at some time instant, their velocities are modified to space them apart without affecting the total momentum. 


\section{Numerical Examples}

\subsection{Sloshing waves in tanks}

Sloshing is the motion of a liquid with free surface in a partially filled liquid container. The phenomenon is often associated with many engineering problems, and one example of application is transport of LNG in tanks on ships.

For a rectangular tank, the natural frequency of the liquid sloshing can be estimated from linear theories ${ }^{26}$ by the following equation,

$$
\omega_{m}=\sqrt{g \frac{m \pi}{L} \tanh \left(\frac{m \pi}{L} d\right)}
$$

where $d$ is the depth of water in the tank, $L$ the length of the tank and $g$ the acceleration due to gravity. Only the fundamental frequency $(m=1)$ is significant for marine engineering application ${ }^{26}$.

\subsubsection{Experiments}

Figure 1 shows the experimental setup. A tank was mounted on a shake table which was subjected to lateral displacement excitation. The displacement of the shake table was measured by a displacement transducer mounted on the shake table. All the output signals were sent to a digital oscilloscope where data can be acquired and stored. The dynamic motion of the fluid was recorded by a high speed camera located in front of the tank.

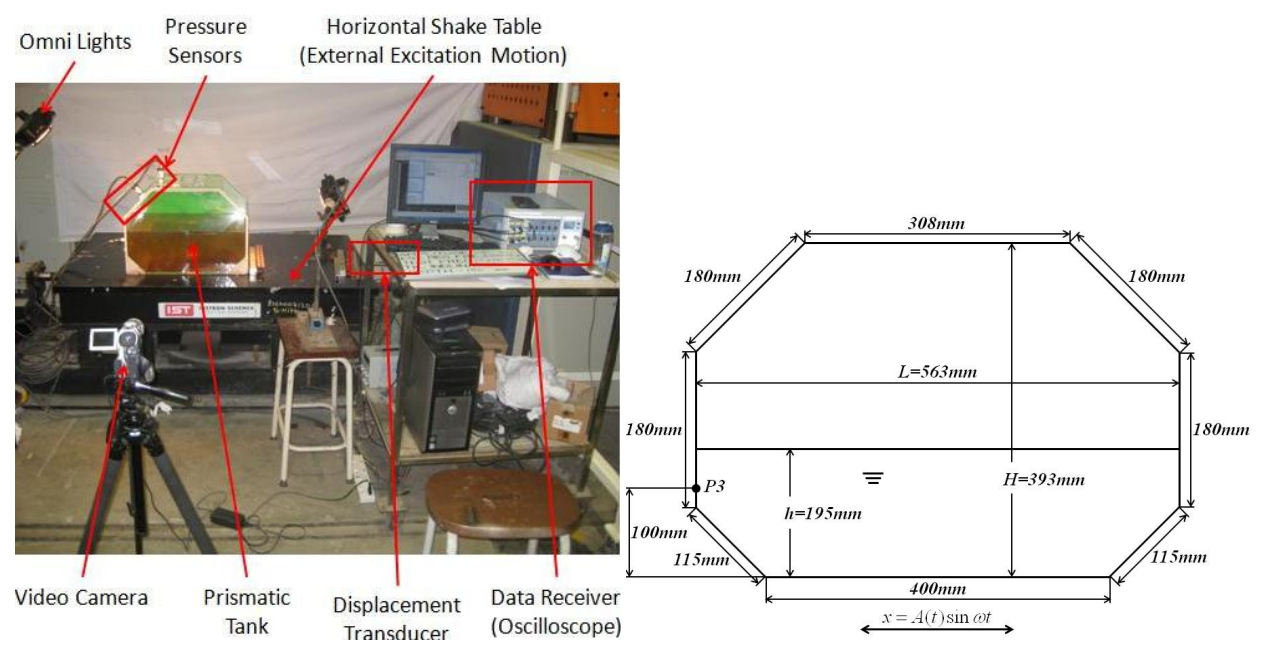

Fig. 1. Experimental setup.

Fig. 2. Definition of parameters for the prismatic tank.

The water tank used in the sloshing experiments is schematically shown in Figure 2. The tank with length $L$ and height $H$ was filled with water up to the filling depth $d$. A 
pressure sensor was used to measure water pressure on one side wall, which is P3 shown in the figure.

In our experiments, the shake table generates unidirectional lateral displacement which is sinusoidal and governed by $x=A \sin (\omega t)$, where $A$ is the amplitude of excitation and $\omega$ the excitation frequency. In order to avoid the big initial impulse, a ramping function shown in Eq. (17) is adopted for the shake table motion.

$$
A(t)=\left\{\begin{array}{l}
0.0005 t, \quad 0 \leq t<10 \\
0.005, \quad t \geq 10
\end{array}\right.
$$

When the excitation frequency is equal to the natural frequency of the liquid in the tank, resonant sloshing occurs. Since Eq. (16) is based on linear wave theory while the violent sloshing is highly nonlinear and the computational domain is not rectangular, the calculated $\omega_{0}$ may not be the exact natural frequency of the sloshing system.

\subsubsection{Experimental and numerical results}

Chen et al. ${ }^{27}$ and Lloyd's Register ${ }^{28}$ have pointed out that the sloshing is most severe when the filling depth (d) is from nearly $50 \% \mathrm{H}$, to $70 \% \mathrm{H}$, where $H$ is the tank height. In our experiment, $50 \% H$ is studied with excitation frequency $\omega=\omega_{0}$.

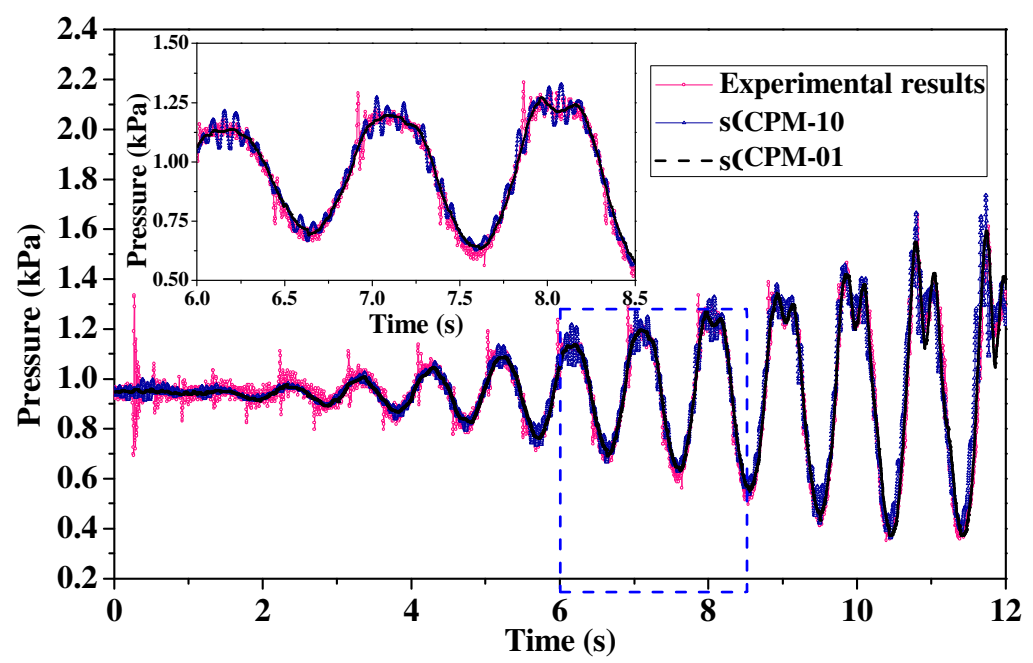

Fig. 3. Comparison of pressure history at excitation frequency of $1.0 \omega_{0}$.

The pressure history at Point P3 using CPM is shown in Figure 3 with the comparison of experimental results. As can be seen, the pressure amplitudes in experiment increase with time. The pressure amplitude at about $11.7 \mathrm{sec}$ is about $1.6 \mathrm{kPa}$, i.e. $70 \%$ larger than the hydrostatic pressure (about $0.929 \mathrm{kPa}$ ). The numerical results agree quite well with the experiments in terms of amplitudes and phase. The local pressure fluctuations are 


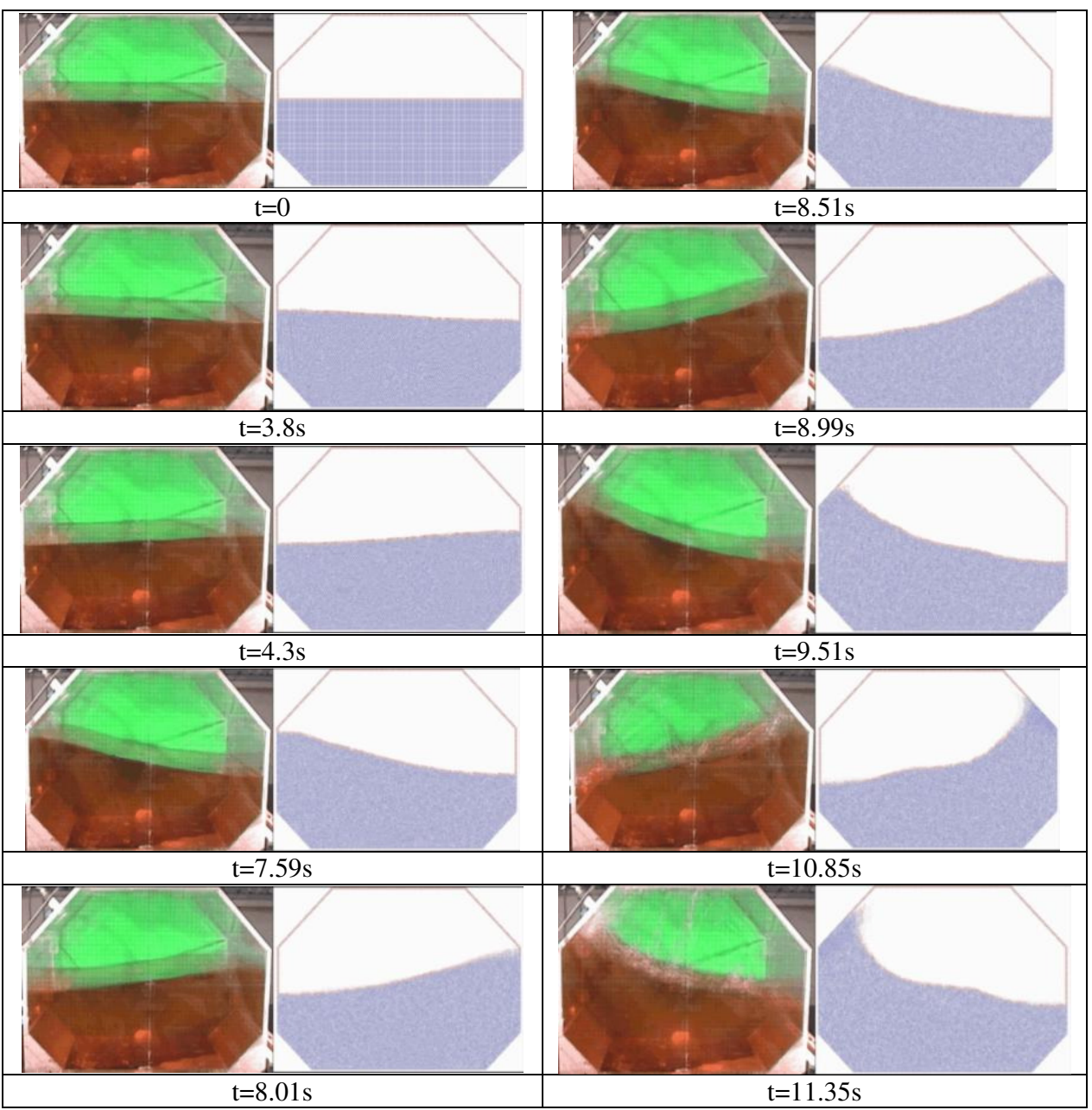

Fig. 4. Wave profiles by CPM-01 compared to experiment at excitation frequency $1.0 \omega_{0}$.

more obvious in the results of CPM-10 especially near peaks. On contrary, CPM-01 has shown smoother pressure results and agrees better with the experiment.

The wave profiles at different time instants simulated using CPM-01 are shown in Figure 4. As can be seen, the numerical results compares well with experiment. Standing waves are observed, in which the wave amplitude becomes larger and larger with time. At about $8 \mathrm{~s}$, the wave begins to touch the chamfer knuckle of the prismatic tank. After about $9.5 \mathrm{~s}$, breaking waves occur, i.e. the wave moves along the chamfer and then fall down under gravity. Even when violent sloshing occurs, the simulation results agree well with the experiments. At 11.35s, slight differences between the experiment and numerical 
results may be caused by the $3 \mathrm{D}$ motion in the experiment, while $2 \mathrm{D}$ simulation is conducted in the numerical study.

\subsection{Breaking wave impacts in tsunami}

In this section, breaking wave impact is simulated. The numerical case is studied by Khayyer and Gotoh ${ }^{29}$. Experiments were carried out in wave flume by Goda and Fukumori $^{30}$ in 1972 and Bullock et al. ${ }^{31}$ in 2007. A schematic sketch of the experimental and numerical domain is shown in Figure 5. Three pressure sensors at location a, b and c were used to measure the impact pressures at different locations. The wave parameters are set the same to the abovementioned studies. This case can be considered as a small scale tsunami example.

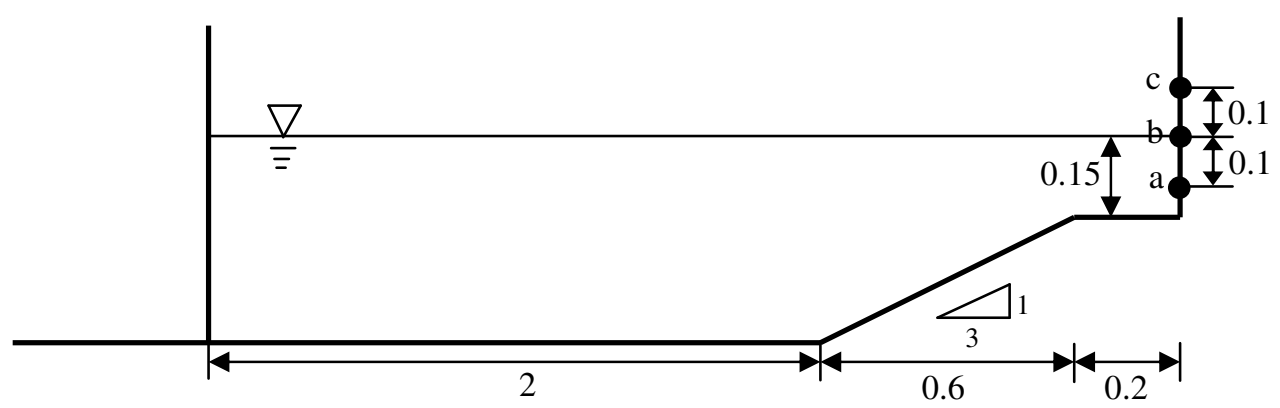

Fig. 5. A Sketch of the study domain.

Figures 6-8 show the comparison of the pressure histories of the three pressure sensors $\mathrm{a}, \mathrm{b}$ and $\mathrm{c}$ respectively. As can be seen all the three figures, the CPM solutions agree well with the experimental results. Both the numerical results and the experiments show that there are two pressure peaks when the wave impact on the wall. Similar to the CMPS-HS and CMPS-HMS results by Khayyer and Gotoh ${ }^{29}$, the CPM results also show small fluctuations. But the CMPS-HS and CMPS-HMS solutions has included hybrid source terms in order to reduce the pressure fluctuation. In our numerical results, CPM10 is adopted, which means no hybrid source term is incorporated. The small fluctuations will be minimized if smaller $\gamma$ is used. Here we don't investigate different $\gamma$ values as the original CPM solutions agree fairly well with the experiments already.

As we can see in Figure 6, the static water pressure is smaller in the numerical results compared to the experiments. This may be because the wave generation method in the numerical study is not the same as in the experiments. In our numerical simulation, the wave generator moves backward first. This causes the water lever reduce before the wave impact the right wall. 


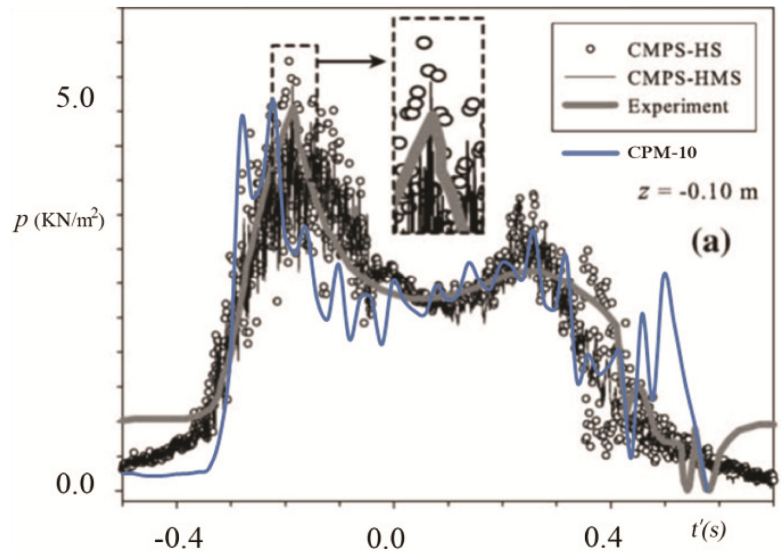

Fig. 6. Comparison of pressure history at sensor a.

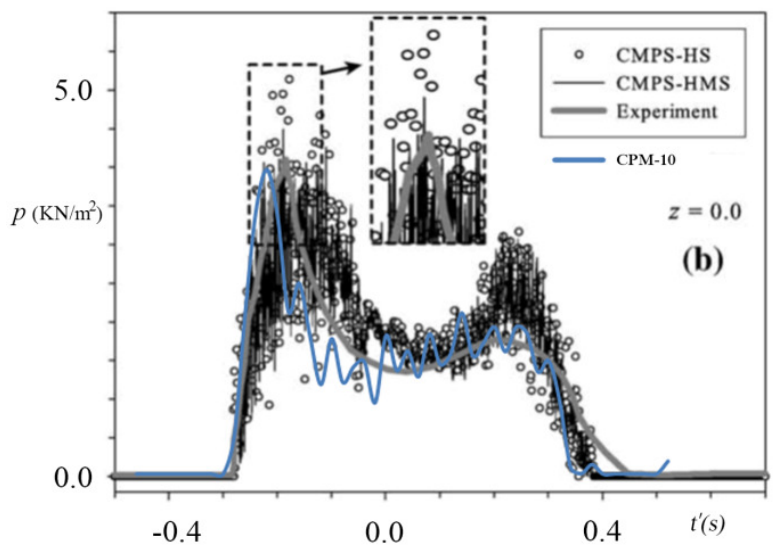

Fig. 7. Comparison of pressure history at sensor $b$.

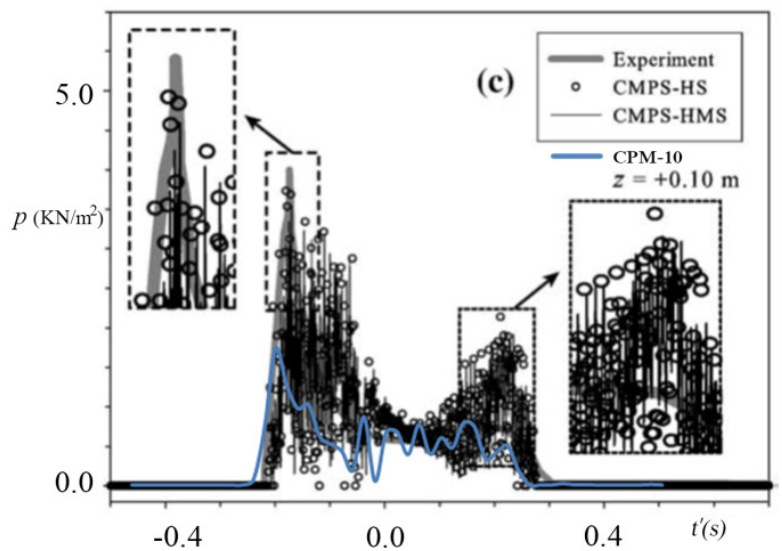

Fig. 8. Comparison of pressure history at sensor $\mathrm{c}$. 
From Figures 7 and 8 we can see that the CMPS results overestimate the impact pressures, especially at the second pressure peak. The CPM solutions, however, agree well with the experimental measurements except at pressure sensor c. The first peak of the CPM solutions is smaller than the experiments. This may be due to the particle resolution in CPM is not high enough. The sensor $\mathrm{c}$ is the one above the static water level. It measures water pressure when the wave climbs to this point and hit the wall. If the particles are not fine enough, few particles will pass by and the pressure computed may be not accurate.

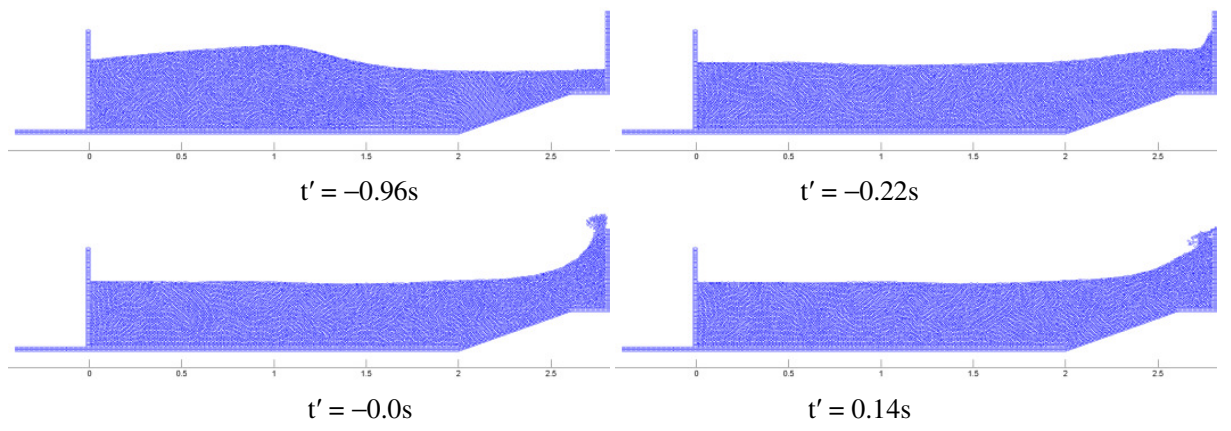

Fig. 9. Wave profiles at four time instances.

The wave profiles at several time instances are shown in Figure 9 with $\mathrm{t}^{\prime}=\mathrm{t}-\mathrm{t}^{\mathrm{mr}}\left(\mathrm{t}^{\mathrm{mr}}\right.$ is the time of maximum run-up). The wave propagation process is demonstrated. The water impact on the wall and fall back to the main water body. Since no published results are available, only the first impact is shown for illustration.

\section{Conclusions}

In this chapter, the CPM is applied to simulate breaking waves in tsunami and sloshing in prismatic tank. The CPM is shown to be capable of simulating violent wave breaking impact. The numerical solutions agree well with published results. Experiments are conducted using a shake table to study violent water sloshing. Comparison between the numerical and experimental results shows that the proposed numerical method CPM is robust in modeling free surface flow problems, especially when wave breaking occurs.

Since in the experiment only translational excitation is applied, multi-degree-offreedom (DOF) excitation is being studied currently. In addition, 3D numerical algorithm is being developed.

\section{Acknowledgments}

The authors appreciate the funding support of the Maritime and Port Authority of Singapore (Grant WBS No. R-302-000-012-490). 


\section{References}

1. S. Koshizuka, A. Nobe and Y. Oka, Int. J. Numer. Methods Fluids 26(1998) 751-769.

2. B. Ataie-Ashtiani and L. Farhadi, Fluid Dyn. Res. 38(2006) 241-256.

3. J. J. Monaghan, Comp. Phys. Commun. 48(1988) 89-96.

4. J. J. Monaghan, J. Comp. Phys. 110(1994) 399-406.

5. S. Koshizuka and Y. Oka, Nucl. Sci. Eng. 123(1996) 421-434.

6. S. D. Shao and E. Y. M Lo, Adv.Water Resour. 26(2003)787-800.

7. A. Souto-Iglesias, L. Perez-Rojas and R. Zamora Rodriguez, Ocean Eng. 31(2004) 1169-1192.

8. J. Fang, A. Parriaux, M. Rentschler and C. Ancey, Appl. Numer. Math. 59(2009) 251-271.

9. H. Y. Yoon, S. Koshizuka and Y. Oka, Nucl. Sci. Eng. 133(1999)192-200.

10. B. Ataie-Ashtiani, G. Shobeyri and L. Farhadi, Fluid Dyn. Res. 40(2008) 637-661.

11. K. Shibata and S. Koshizuka, Ocean Eng. 34(2007) 585-593.

12. D. S. Kershaw, J. Comp. Phys. 26(1978) 43-65.

13. R. Courant, K. Friedrichs and H. Lewy, IBM Journal 1967 215-234 (English translation of the 1928 German original).

14. T. Liszka and J. Orkisz, Comp.Struct. 11(1980) 83-95.

15. X.Y Hu and N. A. Adams J Comput Phys 227(2007) $264-78$.

16. S. J. Cummins and M.Rudman. J Comput Phys 152(1999) 584 - 607.

17. R. Xu, P. Stansby P and D. Laurence. J Comput Phys 228(2009) 6703- 25.

18. M. Tanaka and T. Masunaga. J Comput Phys 229(2010) 4279-90.

19. H. Edelsbrunner, D. G Kirkpatrick and R. Andseidel, IEEE Transactions on Information Theory IT-29(1983) 551-559.

20. H. Edelsbrunner and E. P Mucke, ACM Transactions on Graphic 13(1994) 43-72.

21. S. R. Idelsohn, E. Onate, N. Calvo and F. Del Pin, Int. J. Numer. Methods Eng. 58(2003) 893-912.

22. S. R. Idelsohn SR, E Onate and F. Del Pin, Int. J. Numer. Methods Eng. 61(2004) 64-989.

23. S. R. Idelsohn and E Onate, Comp. Methods Appl. Mech. Eng. 195(2006) 4681-4696.

24. G. A Dilts, Int. J. Numer. Methods Eng. 48(2000) 1503-1524

25. C. G. Koh, M. Gao and C. Luo. Int. J. Numer. Meth. Engng. 89(2012) 1582-1604.

26. T. C. Su, Y. K. Lou, J. E. Flipse and T. J. Bridges, US Department of Transportation, Final Report 1982 MA-RD-940-82046.

27. Y. G. Chen, K. Djidjeli and W. G. Price, Comp. Fluids 38(2008) 830-842.

28. Lloyd's Register. Comparative sloshing analysis of LNG ship containment systems. April 2005.

29. A. Khayyer and H. Gotoh. Coastal Engineering 56(2009) 419-440.

30. Y. Goda and T. Fukumori. Report of the Port and Harbor Research Institute Ministry of Transport, Japan 11(1972) 3-45.

31. G. N. Bullock, G. Obhrai, D. H. Peregrine and H. Bredmose. Coastal engineering 54(2007) 602-617. 